\title{
Oral Anticoagulants in Atrial Fibrillation with Severe or End Stage Chronic Kidney Disease. The Lack of Strong Evidence and the Current Trends Toward a Better Practice. A Mini Review
}

\author{
Rami Riziq Yousef Abumuaileq* \\ Consultant Cardiologist (M.D., Ph.D), Palestine
}

*Corresponding author: Rami Riziq Yousef Abumuaileq, Consultant Cardiologist (M.D., Ph.D.)- Palestinian Medical Services/ Palestinian Ministry of health- Gaza- Palestine and Past doctoral fellow (senior fellow) in interventional cardiac electrophysiology and cardiology department- Tertiary University Hospital of Santiago de Compostela, Spain

\begin{tabular}{|c|c|}
\hline ARTICLE INFO & ABSTRACT \\
\hline Received: 豐 November 18, 2019 & Citation: Rami Riziq Yousef Abumuaileq. Oral Anticoagulants in Atrial Fibrillation with \\
\hline Published: 㓞 November 27, 2019 & $\begin{array}{l}\text { Severe or End Stage Chronic Kidney Disease. The Lack of Strong Evidence and the Cur- } \\
\text { rent Trends Toward a Better Practice. A Mini Review. Biomed J Sci \& Tech Res 23(2)- } \\
\text { 2019. BJSTR. MS.ID.003881. }\end{array}$ \\
\hline
\end{tabular}

\section{Mini Review}

\section{Atrial Fibrillation and Chronic Kidney Disease are Close- ly Related}

Renal impairment is a common comorbidity observed in patients with atrial fibrillation (AF). A bidirectional relationship between $\mathrm{AF}$ and chronic kidney disease (CKD) has long been assumed. The highest incidence of AF is seen in end stage renal disease (ESRD) patients (15.1\%) compared with non-end-stage CKD (9.6\%) and with normal renal function (2.6\%). Several studies concluded that AF may contribute to an accelerated progression of CKD to ESRD independent of other known risk factors. Patients with AF and renal dysfunction are more likely to develop thromboembolic (TE) events compared to those with $\mathrm{AF}$ and normal renal function [1-3]. Moreover, renal dysfunction is an independent predictor of major bleeding and is one of the variables which constitute the known HASBLED and ORBIT bleeding risk scores. Moreover, the prevalence of both comorbidities is common in the elderly/very elderly and with ageing of the world population, their prevalence is expected to increase.

Accurate Estimation of Renal Function is Essential Before Starting Oral Anticoagulation in AF

Patients with $\mathrm{AF}$ are often elderly with multiple comorbidities which need pharmacotherapy of growing complexity, and this makes the reliable estimation of renal function to be undeniably a critical issue. Moreover, the availability of the new non vitamin $\mathrm{K}$ antagonists' oral anticoagulants (NOACs) has renewed the great interest toward the accurate evaluation of renal function in patients with AF. Serum creatinine might not be the best estimate of renal function status particularly in elderly and in over or under weight patients. Glomerular filtration rate (GFR) is poorly inferred from serum creatinine alone. GFR is related inversely and nonlinearly to serum creatinine, age, gender, and race which all affect muscle mass and, in turn, serum creatinine level. The commonly used formulas to estimate GFR include variables to account for serum creatinine variation with age, gender, and race. Several equations were developed to calculate GFR. The commonly used equations to calculate estimated GFR include the Cockcroft-Gault (CG) and Modification of Diet in Renal Disease (MDRD) equation. Other equations used to estimate GFR include the re-expressed MDRD II equation, developed for use with standardized serum creatinine assays; the Mayo Quadratic (Mayo) equation, developed to better estimate GFR in healthy patients with preserved renal function; and the recently developed Chronic Kidney Disease Epidemiology Collaboration (CKD-EPI) equation. Of these five equations, the CKD-EPI equation is known to have the highest accuracy in estimating GFR and is currently recommended for use to estimate GFR by the Kidney Disease: Improving Global Outcomes (KDIGO) clinical practice guidelines [4,5]. 


\section{Vitamin K Antagonists in AF Patients with Severe CKD (Potential Adverse Effects)}

Poor anticoagulation control with warfarin could induce acute kidney injury by causing profuse glomerular haemorrhage and renal tubular obstruction by red blood cell casts in some patients, especially in those with CKD. This condition is known as warfarin-related nephropathy which could be more pronounced if high quality anticoagulation control with Vitamin K antagonists (VKAs) could not be achieved and maintained [6]. VKAs are known to aggravate vascular calciphylaxis which is seen in severe CKD and this in turn might adversely affect the patient renal status and might have additional adverse systemic effects. Calciphylaxis with VKAs could be a result of their inhibition of the vitamin $\mathrm{K}$ cycle and $\gamma$-carboxylation of matrix Gl a protein [7]. Really, poor quality anticoagulation control with VKAs is common in patients with severe CKD. The proportion of time in therapeutic range (TTR) $\geq 60 \%$ decreased in severe CKD and only $21 \%$ of patients on dialysis achieving TTR $\geq 60 \%$. After multivariate adjustment, the magnitude of TTR reduction increased with increasing CKD severity. These findings have implications for more intensive warfarin management strategies in severe CKD or alternative therapies such as NOACs $[8,9]$.

Really, the support to use VKAs in severe CKD came mainly from the findings of large retrospective studies (i.e., not from randomized trials), which have shown VKAs to offer protection from cardiovascular events without increasing bleeding in severe CKD [10]. However, in a recent meta-analysis, VKAs did not offer reduction in deaths, ischemic events, or strokes but increased the incidence of major bleeding in dialysis patients [11]. In addition due to the lack of convincing efficacy and more concern about potential harm in patients with ESRD or dialysis, warfarin has been implicated in the accelerated decline of renal function either through parenchymal micro-bleeds particularly in the setting of poor quality anticoagulation control with VKAs and/or due to aggravation of vascular calcifications with VKAs. A recently published retrospective study on a large cohort of Medicare beneficiaries in the United States of America has demonstrated that apixaban use in AF patients with ESRD on dialysis could be associated with a lower risk of major bleeding compared with VKAs, and the standard $5 \mathrm{mg}$ twice a day dose of apixaban was associated with reductions in thromboembolic and mortality risk [12].

\section{Severe CKD and ESRD in the Landmark Randomized Tri- als of NOACs}

Patients with GFR $<30 \mathrm{~mL} / \mathrm{min}$ in the dabigatran- RELY trial, rivaroxaban- ROCKET AF trial and edoxaban- ENGAGE AF trial and patients with GFR $<25 \mathrm{~mL} / \mathrm{min}$ in apixaban-ARISTOTLE trial were excluded from the pivotal clinical trials of NOACs. This could explain the gap of evidence regarding oral anticoagulation in severe CKD and ESRD. In subgroup analysis of the ARISTOTLE trial, Apixaban was more effective than warfarin to prevent stroke and systemic embolism in the three distinct subgroups of renal function (i.e., GFR
$>80$, GFR > 50-80 and GFR $25-50 \mathrm{~mL} / \mathrm{min}$ ). Patients with the moderate CKD (i.e., GFR $<50 \mathrm{ml} / \mathrm{min}$ ) benefitted from the greatest reduction of major bleeding on apixaban compared to warfarin [13].

\section{Oral Anticoagulants in AF Patients with Severe CKD and ESRD (The Current Practice Guidelines)}

The European Society of Cardiology (ESC) guidelines prefer NOACs over VKAs for the prevention of TE event in AF. The 2018 ESC practical guidelines recommend the use of reduced dose of NOACs [rivaroxaban (15mg 0.D.), apixaban (2.5mg B.D.) and edoxaban (30mg O.D.) for AF patients with high TE risk and severe CKD (i.e., GFR, 15- $29 \mathrm{~mL} / \mathrm{min}$ ). According to the ESC practical guidelines, dabigatran should not be used in patient with severe CKD (i.e., GFR 15- $29 \mathrm{ml} / \mathrm{min}$ ). In the current practical ESC guidelines, all the four NOACs should not be used in patients with ESRD or dialysis (i.e., GFR $<15 \mathrm{ml} / \mathrm{min}$ ), so the ESC guidelines might still support VKAs (international normalized ratio: 2-3) in AF patients with high TE risk and ESRD [14]. On the other hand, the Food and Drug Administration (FDA) in the United States of America approved reduced dose dabigatran $75 \mathrm{mg}$ twice daily, apixaban $5 \mathrm{mg}$ twice daily (apixaban $2.5 \mathrm{mg}$ twice daily if $>80$ year old or $<60 \mathrm{~kg}$ ) and rivaroxaban $15 \mathrm{mg}$ once daily in patients with severe CKD (i.e., GFR 15 - 29mL/ min); this were mainly based on pharmacological modelling data. The recent 2019 American Heart Association/ American College of Cardiology AHA/ACC focused updates have stated that for AF patients with high TE risk and ESRD, it might be reasonable to prescribe VKAs or apixaban for oral anticoagulation (class II b) [15].

\section{Optimal Follow Up Intervals for Renal Function Assess- ment in CKD}

The current ESC practice guidelines and experts opinion proposed a reasonable follow up intervals for renal function assessment which depends on obtaining the baseline GFR and divide it by 10 (e.g., in a patient with baseline GFR of $40 \mathrm{ml} / \mathrm{min}$, divide 40 by 10 , so his renal function should be assessed every 4 months- this could be more frequent in case of acute worsening conditions) $[14,16]$.

\section{Lack of Strong Evidence and A Need for Controlled Ran- domized Trials}

Our current practice regarding the use of oral anticoagulants in AF patients with ESRD is supported mainly by the findings of retrospective studies and not by sizable controlled randomized trials. Evidence might still be insufficient to recommend widespread use of VKAs or NOACs to improve clinical outcomes in patients with ESRD or dialysis dependent patients. Currently, there are three ongoing randomized trials designed to address this dilemma like: RENAL-AF trial, which is conducted to compare apixaban with warfarin in participants with haemodialysis-dependent ESRD and AF; AXADIA trial which aims to evaluate apixaban versus VKAs (phenprocoumon) in haemodialysis patients and the AVKDIAL trial to investigate the efficacy of oral anticoagulation with warfarin versus 
no anticoagulation in patients on dialysis with ESRD and AF. The related scientific communities are still waiting the publication of the final results of these randomized trials which could aid toward the best management strategy of this group of high-risk patients.

\section{Practical Points for Proper Use of Oral Anticoagulants to Improve the Outcomes in AF patients with CKD}

a) Before starting oral anticoagulants, accurate evaluation of the patient's renal function status using GFR or creatinine clearance is necessary.

b) With regard to the use of oral anticoagulants, patients with AF and severe CKD or ESRD should be flagged as a highrisk group and should have more follow up visits than others.

c) A close collaboration between the cardiologist and the nephrologist should be maintained (a multidisciplinary team if possible) for proper decision making and suitable management plan of patients with AF and severe CKD or ESRD.

d) The doctors should follow the dose reduction protocol of NOACs according to the estimated GFR of the patient (as per practical guidelines).

e) For patients who are taking VKAs, continuous efforts should be directed to maintain high quality anticoagulation control with TTR $>65 \%$.

f) Assess proper adherence and carefully check the patient's medication list for potential adverse drug -drug interactions during each follow up visit.

g) Ensure the best management of associated comorbidities (e.g., hypertension, gastrointestinal disease, connective tissue disease etc...) multidisciplinary team is advisable.

h) Optimal follow up intervals for renal function assessmentdepending on baseline GFR, complete blood count and liver function test.

\section{Acknowledgement}

None.

\section{Conflict of Interest}

No conflict of interest.

\section{References}

1. Soliman EZ, Prineas RJ, Go AS, Xie D, Lash JP, et al. (2010) Chronic kidney disease and prevalent atrial fibrillation: The Chronic Renal Insufficiency Cohort (CRIC). Am Heart J 159(6): 1102-1107.

2. Bansal N, Fan D, Hsu CY, Ordonez JD, Marcus GM, et al. (2013) Incident atrial fibrillation and risk of end-stage renal disease in adults with chronic kidney disease. Circulation 127(5): 569-574.
3. Watanabe H, Watanabe T, Sasaki S, Nagai K, Roden DM, et al. (2009) Close bidirectional relationship between chronic kidney disease and atrial fibrillation: the Niigata preventive medicine study.Am Heart J 158(4): 629-636.

4. Levin A, Stevens PE (2014) Summary of KDIGO 2012 CKD Guideline: behind the scenes, need for guidance, and a framework for moving forward. Kidney Int 85(1): 49-61.

5. Abumuaileq RRY, Abu Assi E, Lopez Lopez A, Sergio Raposeiras Roubin, Moisés Rodríguez Mañero, et al. (2015) Renal function assessment in atrial fibrillation: Usefulness of chronic kidney disease epidemiology collaboration vs reexpressed 4 variable modification of diet in renal disease. World J Cardiol 7(10): 685-694.

6. Glassock RJ (2019) Anticoagulant-Related Nephropathy. CJASN 14(6): 935-937.

7. Galloway PAG, El Damanawi R, Bardsley V (2015) Vitamin K Antagonists Predispose to Calciphylaxis in Patients with End-Stage Renal Disease. Nephron 129(3): 197-201.

8. Yang F, Hellyer JA, Than C, Ullal AJ, Kaiser DW, et al. (2017) Warfarin utilisation and anticoagulation control in patients with atrial fibrillation and chronic kidney disease. Heart 103(11): 818-826.

9. Abumuaileq RRY, Abu Assi E, Raposeiras Roubin S, López López A, Redondo Diéguez A, et al. (2015) Evaluation of SAMe-TT2R2 risk score for predicting the quality of anticoagulation control in a real-world cohort of patients with non-valvular atrial fibrillation on vitamin-K antagonists. Europace 17(5): 711-717.

10. Bonde AN, Lip GYH, Kamper AL, Hansen PR, Lamberts M, et al. (2014) Net clinical benefit of antithrombotic therapy in patients with atrial fibrillation and chronic kidney disease: a nationwide observational cohort study. J Am Coll Cardiol. 64(23): 2471-2482.

11. Nochaiwong S, Ruengorn C, Awiphan R, Dandecha P, Noppakun K, et al. (2016) Efficacy and safety of warfarin in dialysis patients with atrial fibrillation: a systematic review and meta-analysis. Open Heart 3(1): e000441.

12. Siontis KC, Zhang X, Eckard A, Bhave N, Schaubel DE, et al. (2018) Outcomes associated with apixaban use in end-stage kidney disease patients with atrial fibrillation in the United States. Circulation 138(15): 1519-1529.

13. Hohnloser SH, Hijazi Z, Thomas L, Alexander JH, Amerena J, et al. (2012) Efficacy of apixaban when compared with warfarin in relation to renal function in patients with atrial fibrillation: insights from the ARISTOTLE trial. Eur Heart J 33(22): 2821-2830.

14. Steffel J, Verhamme P, Potpara TS, Albaladejo P, Antz M, et al. (2018) The 2018 European Heart Rhythm Association Practical Guide on the use of non-vitamin $\mathrm{K}$ antagonist oral anticoagulants in patients with atrial fibrillation. Eur Heart J 39(16): 1330-1393.

15. Craig T January, Samuel Wann L, Hugh Calkins, Lin Y Chen, Joaquin E Cigarroa, et al. (2019) 2019 AHA/ACC/HRS Focused Update of the 2014 AHA/ACC/HRS Guideline for the Management of Patients With Atrial Fibrillation: A Report of the American College of Cardiology/American Heart Association Task Force on Clinical Practice Guidelines and the Heart Rhythm Society in Collaboration With the Society of Thoracic Surgeons. Circulation 140: e125-e151.

16. Gremmel T, Niessner A, Domanovits H (2018) Non-vitamin K antagonist oral anticoagulants in patients with an increased risk of bleeding. Wien Klin Wochenschr 130(23-24): 722-734. 
ISSN: 2574-1241

DOI: 10.26717/BJSTR.2019.23.003881

Rami Riziq Yousef Abumuaileq. Biomed J Sci \& Tech Res

(c) (P) This work is licensed under Creative Commons Attribution 4.0 License

Submission Link: https://biomedres.us/submit-manuscript.php

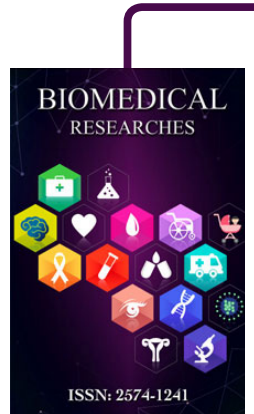

Assets of Publishing with us

- Global archiving of articles

- Immediate, unrestricted online access

- Rigorous Peer Review Process

- Authors Retain Copyrights

- Unique DOI for all articles

https://biomedres.us/ 